\title{
The social epidemiology and construction of risk for Fetal Alcohol Syndrome in Native American communities
}

\author{
Brian P Mangum \\ Fiji National University, Suva, Fiji
}

Correspondence: Brian P Mangum, Fiji National University, Suva, Fiji,Tel 6913202480,Email epidemiology.doc@gmail.com

Received: April 19, 2018 | Published: April 20, 2018

Copyright@ 2018 Mangum. This is an open access article distributed under the terms of the Creative Commons Attribution License, which permits unrestricted use, distribution, and reproduction in any medium, provided the original author and source are credited.

\begin{abstract}
Fetal alcohol syndrome (FAS) is characterized by craniofacial dysmorphology, growth deficiencies, and central nervous system dysfunction. The results of FAS are generally mild to moderate retardation, as well as social dysfunction andlack of social attainment. FAS is entirely preventable by not consuming alcohol during pregnancy. Despite this, FAS remains a factor within the general population, with abnormally high rates among Native Americans. This monograph explores the ways in which sociocultural factors play a role in the construction of FAS as a threat in the Native American community, through an examination of FAS as a public health intervention. By examining the determinants of FAS as cultural factors from the standpoint of social epidemiology, including those underlying economic and political factors that place certain ethnic and cultural groups in a state of disenfranchisement, we are able to see how public health interventions that examine such factors can meet immediate clinical demand as well as address long-term cultural stigma and disenfranchisement. Programs that wish to address FAS in Native American communities must address the lack of community resources, such as alcohol abuse counseling, needed to support educational efforts. In addition to this, long-term studies must address how to effectively implement such programs, as well as addressing underlying economic needs that have been shown to be related to alcohol abuse.
\end{abstract}

Keywords: Fetal Alcohol Syndrome, FAS, Native Americans, social epidemiology, risk

\section{Introduction}

Fetal alcohol syndrome (FAS) is a health concern across all socioeconomic and cultural/ethnic groups. ${ }^{1}$ However, among certain ethnic and cultural groups, FAS, with the concurrent costs to both the individual and society, is considered a greater problem. This is particularly true among the Native American population, which has FAS rates $33 \%$ higher than the Caucasian population. ${ }^{1} \mathrm{FAS}$ is a condition that is entirely preventable by not drinking during pregnancy. ${ }^{1}$ Given the preventable nature of FAS, the question arises of what are the underlying reasons that Native American women continue to drink during pregnancy, given the life-altering detriments of FAS to the unborn child. Clearly, there are factors at work more complicated than simply stating that FAS can be prevented by not drinking during pregnancy.

That is what this monograph seeks to address, or what are the underlying sociocultural reasons that Native American women drink during pregnancy, thus exposing their unborn child to the risks of FAS. In so doing, this monograph will explore FAS among Native Americans from the perspective of a public health intervention based on the social epidemiology of the disease. In this way, not only will the monograph be able to explore the sociocultural construction of FAS in the Native American community through a discussion of the various determinants of FAS, and the social construction of risk perception of such, but will also be able to develop a practical framework for addressing such problems that is culturally appropriate. In so doing, the following points will be discussed: (a) what is FAS, including the long-term effects of such; (b) what is the epidemiology of FAS in the general population, as well as the Native American population (c) what are the sociocultural and behavioral determinants of FAS in the Native American community, and how can these determinants be addressed through a culturally appropriate public health intervention; (d) this monograph will conclude with recommendations for further study of FAS in the Native American community as well as recommendations for implementing prevention programs.

\section{Etiology of fetal alcohol syndrome}

To begin the discussion of the sociocultural construction of FAS in Native American Communities, the monograph will first discuss what FAS is, and what the long-term effects of the disorder are. FAS is a neurodevelopmental and physical syndrome that is characterized by a triad of symptoms, including "...craniofacial dysmorphology, growth deficiencies, and central nervous system dysfunction". ${ }^{2}$ This triad of symptoms is used to make the diagnosis of FAS, although reliance upon morphology and phenotype among infants is the prime diagnostic feature, ${ }^{3}$ particularly among newborns. Later in life, FAS may be diagnosed using other factors such as cognitive ability as well as craniofacial features. ${ }^{4}$ Regardless of diagnosis, FAS is the result of exposure of the fetus to alcohol in utero, and results in lifelong morbidity, particularly characterized by developmental delay as well 
as physical malformation. ${ }^{2}$ Studies indicate that FAS can be caused by as little as 1-ounce of absolute alcohol per day in utero; however, most cases of FAS are linked to much greater intake of alcohol, usually around $60-75 \mathrm{ml}$ of absolute alcohol consumption per day during the pregnancy. ${ }^{1,2}$ Accordingly, the CDC states that there is no determined safe level of alcohol consumption during pregnancy, ${ }^{1}$ with the Royal College of Obstetrician and Gynaecologists stating that women should limit consumption of alcohol to one standard drink per day. ${ }^{5}$

In terms of diagnosis, it is interesting to note that the degree to which the child with FAS is developmentally delayed is related to the degree of craniofacial abnormalities. ${ }^{2}$ That is to say, that the greater the craniofacial abnormalities, the greater the developmental and neurocognitive delays the child will possess. Developmental specialists often use the degree of craniofacial abnormalities to determine the level of intervention needed, as well as to estimate the degree to which the child's neurocognitive abilities can be trained. ${ }^{3,4}$ Still, there is caution in the overuse of such phenotypical diagnosis to the detriment of other testing, as FAS can mimic other alcohol related syndromes. Particularly, it should be noted that FAS is different from fetal alcohol effect (FAE), in which a child who has been exposed to alcohol during pregnancy suffers the craniofacial abnormalities but not the cognitive deficits. ${ }^{2}$ The phenotypical expression of FAS is characterized by microcephaly, a flat mid face, thin upper lip, short nose, minor ear abnormalities, low nasal bridge and others. ${ }^{6,7}$ In addition to the underlying neurodevelopmental delays that such physical manifestations represent, such physical malformations also represent a stigmatized view of both the patient of himself as well as the world to the patient, resulting in a cycle of both physical, intellectual, and emotional difficulty which the patient must bear throughout their lives.

The long-term effects of FAS are related primarily to physical and developmental abnormalities. The greatest developmental disability related to FAS is mild to moderate retardation, including difficulty with arithmetic, inattention, poor concentration, memory deficit, and poor judgment.Other problems include those related to motor deficits, such as fine motor skills, and behavioral problems, including attentiondeficit hyperactivity disorder (ADHD) and maladaptive behavior as adults. ${ }^{2}$ There are also problems related to growth, including deficiencies in head circumference, as well as microcephaly, and substandard height and weight both as infants, adults and children. ${ }^{2,8}$ In addition to this, children diagnosed with FAS may also suffer from cardiac murmurs, heart vessel abnormalities, skeletal abnormalities and hernias of the diaphragm, groin and umbilicus. ${ }^{2}$

Given such, the long-term prognosis of the child diagnosed with FAS is not positive. Growth and craniofacial abnormalities do become less obvious over time, and the weight to height proportion may improve as the child grows older, ${ }^{2}$ thus improving the sociocultural conditions under which the patient with FAS may learn to survive if not thrive in contemporary culture. However, other physical malformations, such as short stature and microcephaly are persistent conditions that do not generally improve. ${ }^{8}$ Neurodevelopmental handicaps do not generally improve with time, and most individuals with FAS will have lifelong cognitive handicaps preventing them form obtaining educational and social attainment with most "...failing to achieve independent income or housing", ${ }^{2}$ thus depriving them of the traditional paths to social status attainment within the larger culture. However, and while there have been no studies done to examine such, it would be interesting to examine self and community conceptualizations of the individual with FAS among Native American communities to determine of differences exist between such and mainstream American culture.

\section{Descriptive epidemiology of fetal alcohol syndrome:native americans versus the general population}

Having discussed what FAS is, including the long-term effects of such, this monograph will now turn to a brief discussion of the epidemiology of FAS among the general and Native Americans populations. The rates of FAS in the general population vary widely according to various demographic factors, such as ethnicity and geography. This is true among the general population of the United States, as well as ethnic groups, such as Native Americans, where there are varying rates of FAS present among different tribal groups. This highlights a specific point, in that it is difficult when engaging in a cultural study such as this to determine the extent of a health problem, given that contemporary statistics tend to differentiate little with regard to such factors as sub-groups within ethnic categories such as Native Americans. According to the CDC, the rates of FAS range from 0.2 to 1.5 per 1,000 births in the general population. ${ }^{9}$ Demographically, Asian Americans have the lowest rates of FAS at 0.3 per 10,000 births, with Hispanics having a rate of 0.8, Caucasians 0.8, African Americans 6.0, and Native Americans the highest demographic rate at 29.9 cases per 10,000 births. $^{10}$

The rate of FAS among different tribal groups varies widely as well, ranging from 13 to 103 per 10,000 births in the American Southwest. ${ }^{11}$ Among the Navajo and Pueblo tribes, the rate of FAS is more similar to the overall rate for the United States, while among the Southwest Plains Native Americans there is a much higher rate of one per every 102 live births. ${ }^{12}$

\section{Social epidemiology of the determinants and risk constructs of fetal alcohol syndrome}

Thus, it can be seen that among the Native American there is a varying rate of FAS present in the population, that might be understood better from a sociocultural standpoint, and include issues related to economics, education, access to healthcare and so forth. For instance, among the Navajo tribes, where there is greater economic development, there are also fewer reported cases of FAS than other tribes. ${ }^{12}$ Given this, we can turn to an understanding of such sociocultural and economic factors as determinants of health as related to FAS, and thus examine them from the model of a public health intervention based in the social epidemiology of such. Public health interventions are based upon understanding the underlying lying causes of a health problem in terms of direct and indirect determinants. Such determinants can be seen as the risk factors that influence the level of health problems. ${ }^{13}$

For instance, when considering the health problem of high blood pressure, one could state that obesity, diet, and cigarette smoking are all determinants of the problem, as well as being behavioral in nature. Determinants are further impacted by direct contributing and indirect contributing factors that influence the level of the determinant. Indirect contributing factors are those that affect the determinant by affecting the direct contributing factor. Returning to our example of high blood pressure, a direct contributing factor of the determinant of obesity would be sedentary lifestyle or lack of activity, 
with an indirect contributing factor being the amount of television that Americans view. In so doing, public health interventions rely upon a series of steps with the ultimate goal in mind of addressing or lowering the risk factor. Public health programs designed to address health issues do so by addressing these determinants and contributing factors, thus addressing the underlying issues that have resulted in the health problem. These steps are activities, process objectives, impact objectives and outcome objectives. ${ }^{13}$

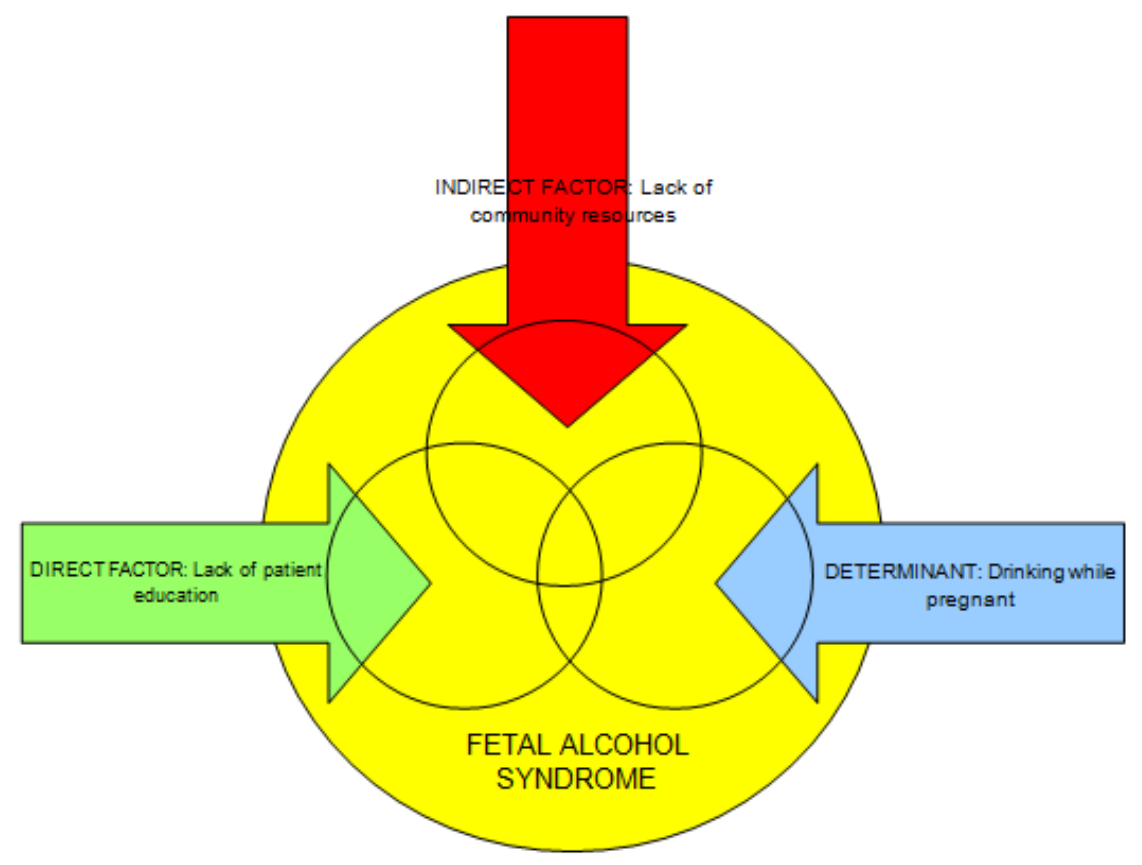

Figure I Graphical representation of the sociocultural construction of FAS in Native American communities.

However, such program must be certain that the issues they are addressing truly are the underlying sociocultural determinants of the problem, and not merely symptoms of a larger systemic problem. For instance, programs that provide condoms to sex workers in Africa do not address the underlying sociocultural inequity that does not grant sex workers the ability to demand condom use, as well as the economic reasons why women must turn to the sex trade to support themselves while being placed at greater risk for sexually transmitted infections (STI) such as HIV/AIDS. It is important to understand, that properly done, the choice of determinants, which are generally behaviorallybased, will expose the underlying sociocultural basis of a healthcare problem, including cultural bias, stigma, and other undesirable attributes of any culture which results in the disenfranchisement of cultural and social groups. Given this, well-designed public health interventions go beyond simply addressing health needs within a specific population, to targeting and alleviating social and cultural injustice in the form of health disparities, and thus meet the larger goals of the growing and influential field of social epidemiology. Such health disparities are generally not defacto, in that they are the product of social and legislative policies designed to result in such, but do represent underlying economic and cultural bias. For instance, while programs that cut Medicaid funding for uninsured individuals and families are not targeted with a specific malignancy towards such groups, and rather may be economically based in times of deficits and decreased tax revenue, they do have the impact of placing such groups at risk in relation to their socioeconomic status within the larger cultural system.
Given such, addressing the determinants of FAS among the Native American population, must take the form of direct contributing factors, but also indirect contributing factors. Indeed, if the determinant can be seen as the underlying causative factor of the health problem, then the direct and indirect determinants can be seen as the true sociocultural stigma and bias that have resulted in such.

Therefore, in terms of the health problem of FAS in general, including Native American women, there can be only one determinant, or that of drinking while pregnant, leaving us to question why women in Native American communities have such a higher prevalence of drinking while pregnant, or the direct and indirect contributing factors. In terms of direct and indirect contributing factors, this monograph will identify one of each, which will be discussed in further detail, including objectives to deal with such. These include the direct contributing factor of a lack of education on the part of patients as to what FAS is and how it can be prevented, with the indirect factor being a lack of community resources.

Patient education models of FAS prevention in Native American communities have been shown to be effective when combined with other methods, particularly assistance in accessing community service agencies to gain access to alcohol abuse programs ${ }^{14}$ thus underpinning the issue that FAS education can be a useful tool if it is combined with programs to address underlying socioeconomic issues related to risk. Thus, it is theoretically possible that if short term access to resources, such as adequate counseling, are the key components to health education models addressing FAS, then long-term models that address 
educational and employment opportunities in the community could be seen as a way of directly affecting FAS on a community-wide basis. Such short-term assistance could be seen as meeting the process objectives of a health intervention. Process objectives are those activities that change the contributing factor, or in this case, a lack of patient education. However, as stated above, patient education must be coupled with addressing underlying economic issues, which from a behavioral standpoint have been shown to have a direct corollary between alcohol abuse and socioeconomic status. ${ }^{5}$ Therefore, in order to meet the needs of patient education in the community, several steps must be taken. These include educating providers about the need to not only provide culturally appropriate counseling regarding the risks of FAS, but also helping at risk patients access the services they need which will allow the patient education model to be most effective. This would include educating providers about the services available in Native American communities, including drug and alcohol counseling, access to government sponsored housing for pregnant and at-risk women, as well as food assistance, and in the long term the possibility of job training and other educational opportunities. Such services would allow a program to meet its impact objectives, which is a measure of the reduction of the determinant in a specific amount of time. ${ }^{13}$ In this case, the determinant to be reduced is the consumption of alcohol by Native American women who are pregnant or are planning to become pregnant.

However, such process and impact objectives cannot address the direct determinants if there is a lack of community resources to assist with such, particularly in regard to drug an alcohol counseling programs that address the needs of women. Tragically, despite Indian Health Services (IHS), this is often the case in Native American communities, where the true underlying social problem is not a lack of patient education, but a lack of those services that allow patient education to be successful. According to Thomason ${ }^{14}$, the problem is not in a total lack of resources, but rather in a lack of culturally appropriate resources for dealing with alcohol counseling that are directed at women. While IHS does offer alcohol counseling programs, such are generally directed towards men, who have represented the traditional burden of alcohol abuse in native communities in terms of recognition, if not actual practice. ${ }^{2}$ This includes a lack of female counselors, or counseling methods directed at women and their unique place within the Native American community. Furthermore, such is compounded by a lack of recognition for referral by providers, ${ }^{2,15}$ as well as a low rate of referral acceptance by patients. ${ }^{16}$ In such an atmosphere where providers fail to recognize the need for referral for alcohol counseling among Native American women at risk for FAS, and where counseling services are not available that are culturally acceptable in terms of being directed towards women as well as an understanding of FAS, it is clear that patient education alone will not allow women to successfully stop drinking during pregnancy to reduce the risk of FAS.

While such is not a physical lack of resources within the community to help women successfully combat alcohol dependence during pregnancy or for those considering becoming pregnant, there is in fact a psychological absence or barrier between these resources and the women who need them. Therefore, any program designed to intervene in the cycle of FAS in the Native American community will be able to meet the patient education process objective (e.g. lack of patient education) within the framework of the provider, however, in terms of the ultimate goals or the impact and outcome objectives, the program will fail, as the community resources that will allow patient education to be effective are not present. ${ }^{14}$ Therefore, the determinant of drinking while pregnant and the outcome objective of reducing FAS in the Native American community cannot be met.

\section{Conclusions and recommendations}

FAS is an entirely preventable disorder. ${ }^{.}$Yet despite this, the percentage of children born with FAS in Native American communities remains disturbingly high. Given this, public health interventions must be designed that take into account the needs of both the community and the individual when assessing how best to intervene. Programs designed to educate patients regarding the risks of FAS and how to prevent such must be predicated upon community resources, including appropriate alcohol abuse and dependence treatment programs. While IHS has such programs in place, they are primarily geared towards men, who represent the traditional burden of alcohol abuse in Native American communities. Studies have shown that if patient education models are to be successful, then such programs must be in place to provide access to community resources such as alcohol abuse counseling.

Further studies are needed to assess how best to implement patient education models that are integrated with community resources that include not only drug and alcohol abuse counseling, but also resources to address underlying socioeconomic disparities that lead to alcohol abuse in the first place. Such programs that combine not only short-term interventions for those who are at risk for FAS, but also address long term disparities in terms of the availability of economic and educational opportunities for Native American communities are needed. Such studies would help provide one of the key components of public health interventions, or that of evidence based practice.

\section{References and further reading}

1. https://www.cdc.gov/ncbddd/fasd/documents/fas_guidelines_accessible. pdf

2. Entin E. Fetal alcohol syndrome. In: Saunders Manual of Medical Practice. W.B. Saunders; 2000.

3. Astley SJ, Clarren SK. A fetal alcohol syndrome screening tool. Alcohol Clin Exp Res. 1995;19(6):1565-1571.

4. Bagheri MM, Burd L, Martsolf JT, Klug MG. Fetal alcohol syndrome: maternal and neonatal characteristics. J Perinat Med-Off J WAPM. 1998;26(4):263-269.

5. Wilkie S. Global overview of drinking recommendations and guidelines. AIM Dig. 1997;2(Supplement):4.

6. Hagerman RJ. Neurodevelopmental Disorders: Diagnosis and Treatment. Oxford University Press; 1999.

7. Pytkowicz Streissguth A, LaDue RA, Randels SP. A manual on adolescents and adults with fetal alcohol syndrome with special reference to American Indians. 1986.

8. Hay W, Levin M, Sondheimer J, Hayward A. Current Pediatric Diagnosis \& Treatment. 15th ed. New York: McGraw-Hill; 2001.

9. Centers for Disease Control. Alcohol consumption among women who are pregnant or who might become pregnant-United States, 2002. MMWR Morb Mortal Wkly Rep. 2004;53(50):1178.

10. Chavez GF, Cordero JF, Becerra JE. Leading major congenital malformations among minority groups in the United States, 1981-1986. MMWR CDC Surveill Summ Morb Mortal Wkly Rep CDC Surveill Summ. 1988;37(3):17. 
11. US Department of Health and Human Services. Report of the Secretary's Task Force on Black \& Minority Health: Crosscutting Issues in Minority Health. 1985.

12. May PA, Hymbaugh KJ, Aase JM, Samet JM. Epidemiology of fetal alcohol syndrome among American Indians of the Southwest. Soc Biol. 1983;30(4):374-387.

13. Turnock BJ. Public Health: What It Is and How It Works. 3rd ed. Sandbury, MA: Jones \& Bartlett; 2004.

14. Thomason TC. Issues in the treatment of Native Americans with alcohol problems. J Multicult Couns Dev. 2000;28(4):243-252.

15. Cummings NA, Cummings JL, Johnson JN. Behavioral health in primary care: A guide for clinical integration. 1997.

16. Gatchel RJ, Oordt MS. Clinical Health Psychology and Primary Care: Practical Advice and Clinical Guidance for Successful Collaboration. Washington, DC: American Psychological Association; 2003.

17. Abel EL, Sokol RJ. Incidence of fetal alcohol syndrome and economic impact of FAS-related anomalies. Drug Alcohol Depend. 1987;19(1):5170 .

18. Abel EL, Sokol RJ. A revised conservative estimate of the incidence of FAS and its economic impact. Alcohol Clin Exp Res. 1991;15(3):514-524.

19. Barr HM, Streissguth AP. Identifying maternal self-reported alcohol use associated with fetal alcohol spectrum disorders. Alcohol Clin Exp Res. 2001;25(2):283-287.
20. Farmer P. Pathologies of Power: Health, Human Rights, and the New War on the Poor. Berkeley, CA: University of California Press; 2003.

21. Frank RG, McDaniel SH, Bray JH, Heldring M, eds. Primary Care Psychology. Washington, DC: American Psychological Association; 2003.

22. House JS, Umberson D, Landis KR. Structures and processes of social support. Annu Rev Sociol. 1988;14(1):293-318.

23. Klerman LV, Ramey SL, Goldenberg RL, Marbury S, Hou J, Cliver SP. A randomized trial of augmented prenatal care for multiple-risk, Medicaideligible African American women. Am J Public Health. 2001;91(1):105.

24. Klug MG, Burd L. Fetal alcohol syndrome prevention: annual and cumulative cost savings. Neurotoxicol Teratol. 2003;25(6):763-765.

25. Mattison DR, Damus K, Fiore E, Petrini J, Alter C. Preterm delivery: a public health perspective. Paediatr Perinat Epidemiol. 2001;15(s2):7-16.

26. Schneiderman NE, Speers MA, Silva JM, Tomes HE, Gentry JH. Integrating Behavioral and Social Sciences with Public Health. American Psychological Association; 2001.

27. Streissguth AP, O’Malley K. Neuropsychiatric implications and long-term consequences of fetal alcohol spectrum disorders. In: Seminars in Clinical Neuropsychiatry. Vol 5; 2000:177-190. 